\title{
Current Status of Syndesmotic Injuries: Our Understanding Today
}

\author{
Andres Rodriguez-Buitrago ${ }^{1}$, Rodrigo F Pesántez ${ }^{2}$
}

\begin{abstract}
Syndesmotic injuries are common after ankle fractures. Despite the growing number of manuscripts on the fluoroscopic images performing the topic, there is still debate surrounding key aspects of syndesmotic injuries. This review aims to present current concepts about the diagnosis and treatment of these injuries.
\end{abstract}

Keywords: Ankle fractures, Ankle joint, Malleolar fracture, Syndesmosis, Syndesmotic injury. Journal of Foot and Ankle Surgery (Asia Pacific) (2021): 10.5005/jp-journals-10040-1134

\section{BACKGROUND/INTRODUCTION}

Our understanding of the ankle complex, syndesmosis injuries, and significance of syndesmotic instability has evolved in the last 50 years shifting from no treatment at all to fixing all the fractures. In recent years, it has been recognized that not all syndesmotic injuries require stabilization. Still, there are no definitive answers regarding the best treatment option (i.e., operative vs nonoperative, and tight rope vs screw), and hardware removal. Recently, a survey of the Orthopaedic Trauma Association (OTA) members showed a high grade of variability and controversy in the treatment of ankle fractures. ${ }^{1}$ In this review, we aim to present what is currently known about the diagnosis, treatment, and postoperative management of syndesmotic injuries.

\section{Review}

Syndesmotic injuries usually occur after ankle pronation external rotation injuries and can be associated with malleolar fractures. ${ }^{2}$ A better understanding of the ankle and syndesmotic anatomy has allowed the orthopedic surgeon to treat syndesmotic injuries. The medial, lateral, and posterior malleoli are important for ankle stability. Like the pelvis, the ankle can be conceived as a ring and altering its bony or ligament structures in at least two places can lead to instability. ${ }^{3}$

\section{Diagnosis}

Physical exam and clinical signs have not shown to be reliable in diagnosing syndesmotic injuries. ${ }^{3-5}$ Therefore, standard radiographs [anterior-posterior (AP), lateral, and oblique view] should be obtained to evaluate patients with a suspected ankle injury. Well-known X-ray parameters that suggest a syndesmotic injury are tibiofibular (TF) overlap ( $>6 \mathrm{~mm}$ in the AP view and $>1 \mathrm{~mm}$ from the tibial plafond in the mortise view), TF clear space $(<6 \mathrm{~mm}, 1$ $\mathrm{cm}$ above the tibial plafond in the AP and mortise views), and medial clear space. Croft et al. also suggested using the anterior tibiofibular ratio (ATFR) measured on the lateral view as an additional measure that can aid in the diagnosis of a syndesmotic lesion. ${ }^{6}$

Dynamic stability of the ankle should be assessed using either external rotation stress, gravity stress, or weight-bearing (WB) radiographs. It is important to bear in mind that each of these methods has pros and cons, for example, manual stress and WB
${ }^{1}$ Department of Orthopedics and Traumatology, Fundación Santa Fe de Bogotá, Universidad del Rosario, School of Medicine and Health Sciences, Bogotá, Colombia

${ }^{2}$ Department of Orthopedics and Traumatology, Fundación Santa Fe de Bogotá, Bogotá, Colombia

Corresponding Author: Rodrigo F Pesántez, Department of Orthopedics and Traumatology, Fundación Santa Fe de Bogotá, Bogotá, Colombia, Phone: +57 3106883199, e-mail: rpesantez@gmail. com

How to cite this article: Rodriguez-Buitrago A, Pesántez RF. Current Status of Syndesmotic Injuries: Our Understanding Today. J Foot Ankle Surg (Asia Pacific) 2021;8(1):19-23.

Source of support: Nil

Conflict of interest: None

radiographs are dependent on patient's pain tolerance, which can affect its technique. ${ }^{7}$ Obtaining ankle stress radiographs in dorsiflexion and external rotation has shown to predict deltoid ligament disruption. ${ }^{8,9}$ Although reliable, this technique has the potential drawback of increased radiation for the medical personnel and limited reliability due to the patient's pain tolerance., ${ }^{70}$ Gravity stress images have shown to be equivalent to manual stress radiographs while being more comfortable for the patient, additionally, it has been reported to have nearly perfect sensitivity and specificity for deltoid ligament disruption. ${ }^{11-14}$ On the contrary, literature has shown that it can overestimate the need for surgical treatment as it increases medial clear space secondary to plantar flexion of the ankle. , 15,16 Weight-bearing radiographs allow evaluation of stability and congruence of the ankle joint while reducing the need for surgery. ${ }^{3,17,18} A$ recent study concluded that WB radiographs fail to demonstrate rotational instability and deltoid ligament lesion. ${ }^{19}$ Weber et al. evaluated the use of WB radiographs taken 3-10 days post-injury founding that this approach allows for a pain-free evaluation while being reliable in distinguishing those injuries that require surgery without affecting functional results. ${ }^{17}$

In cases in which a syndesmotic lesion is suspected but no definitive diagnosis can be made based on X-rays and clinical history alone, a magnetic resonance imaging (MRI) can be warranted. Literature has shown conflicting results, some studies have shown suboptimal interobserver agreement, although more recent studies 
Table 1: Senior author's preferred method of treatment

\begin{tabular}{ll}
\hline Preoperative evaluation & X-rays/CT scan \\
Intraoperative evaluation & $\begin{array}{l}\text { X-rays/3D intraoperative imaging } \\
\text { if available }\end{array}$ \\
Reduction technique & Open \\
Fixation technique & Trans-syndesmotic screw \\
Postoperative protocol & WB as tolerated \\
Hardware removal & Not recommended \\
\hline
\end{tabular}

have reported that the MRI has high sensitivity and specificity while reducing the rate of false-positive injuries that require surgery. ${ }^{20-25}$ Despite having lower soft-tissue resolution when compared to the $\mathrm{MRI}$, computed tomography (CT) allows the surgeon to evaluate the presence of a diastasis, evaluate the incisura fibularis, and when fractures are present, the articular extension, impaction, and presence of a posterior fragment (Table 1).

In the setting of an ankle fracture, diagnosis of syndesmotic instability can be made intraoperatively under fluoroscopic guidance using either the hook test or external rotation stress test. ${ }^{26}$

\section{Treatment}

Once an ankle fracture or syndesmotic injury is deemed to require surgical fixation, the surgeon must consider the different options to obtain and maintain reduction.

\section{How Do I Obtain an Adequate Reduction?}

It is critical to obtain an adequate reduction of the syndesmosis, this can either be performed via indirect or direct methods. It is important to consider the anatomic variabilities of the incisura fibularis. Boszczyk et al. performed bilateral postreduction CT scans and found that over-compression was common in patients with a deep and disengaged incisura, while version of the incisura may lead to anterior or posterior displacement of the fibula. ${ }^{27}$

A reduction clamp is the simplest method that can be used to reduce the syndesmosis, but adequate positioning is crucial, as a too anteriorly positioned tine can malreduce the syndesmosis translating the fibula and narrowing the incisura anteriorly and vice versa which results in malreduction. Efforts have been made to identify the most accurate clamp position. Clamp placement along the axis of the ankle joint (i.e., tines should be positioned lateral to the malleolar ridge at the fibula and in the central part of the medial cortex in the tibia) has historically been used. ${ }^{28,29}$ To evaluate if clamps were anatomically placed, Phisitkul et al. recommended that no overlap of the clamp tines should be seen in a fluoroscopic mortise view. ${ }^{28}$ Cosgrove et al. described medial tine placement in the anterior third of the tibial line. ${ }^{30}$ In a subsequent study, the trans-syndesmotic axis (TSA) measured in a preoperative CT scan was used as a reference for clamp placement, lower malreduction rates were obtained while positioning the clamp along the TSA axis or $10^{\circ}$ posterior to it. ${ }^{31}$ The use of the TSA has proven to help guide adequate clamp positioning. ${ }^{32}$ In a recently published cadaveric study, Vetter et al. compared reduction with collinear clamps, reduction forceps, and crossing K-wires concluding that reduction could be successfully achieved with either method. ${ }^{33}$ Comparison of these methods to digital reduction revealed the absence of over-compression, although clinical outcomes do not differ when compared to clamp reduction. ${ }^{33,34}$

It is important to keep in mind that malreduction and overcompression are common with these techniques. ${ }^{35-37}$ Therefore,
Sagi et al. recommended a "direct, open visualization of the syndesmosis during reduction maneuver". ${ }^{35}$ Miller et al. reported on 149 patients treated with direct visualization and concluded that even though the reduction was suboptimal in some cases, there was a significant decrease in the rate of malreductions. ${ }^{36}$ Recently, Tornetta et al. proposed using the articular surface as a visual landmark during open reduction aligning it with the anteromedial fibular articular surface, by doing so, alignment is further improved when compared to relying on the incisura alone. ${ }^{38}$

Bringing back the "ring concept", it is important to evaluate if a posterior malleolus fracture is present. The posterior malleolus is the attachment site for the posterior inferior tibiofibular ligament (PITFL). Thus, disruption of the posterior complex can lead to syndesmotic instability. Literature has shown that fragments as small as $10 \%$ can affect stability and, reduction and stabilization of this fragment, could be enough to restore syndesmotic stability. ${ }^{39-41}$ Even after fixation of this fragment has been achieved, it is important to rule out persistent syndesmotic instability.

Irrespective of the technique used, it is important to assess the adequacy of reduction, as it will be mentioned in the following sections.

\section{How Should I Fix It?}

Once reduction has been obtained, the surgeon faces the question of whether to use a static (screw) or dynamic fixation. And, while considering a screw fixation, many questions arise. Several biomechanical and clinical studies have addressed many of the questions and there seems to be no difference in screw size (i.e., 3.5 or $4.5 \mathrm{~mm}$ screws), although resistance to shear stress is better tolerated with $4.5 \mathrm{~mm}$ screws, ${ }^{42-44}$ material (stainless steel or titanium) ${ }_{1}^{45}$ the use of one or two screws, ${ }^{46}$ three or four for cortical, ${ }^{43,45-49}$ and location (trans- or supra-syndesmotic). ${ }^{46,50}$

Advocates of suture-button devices argue that, since the syndesmosis is a mobile joint, a dynamic stabilization should be biomechanically more favorable as it allows movement while preserving reduction. A recently published in a meta-analysis including 11 cadaveric studies, concluded that suture-button fixation is less rigid when compared to screw fixation. ${ }^{51}$ There seems to be no difference among the different configurations (i.e., single, parallel, or divergent). ${ }^{52} \mathrm{~A}$ randomized control study by the Canadian Orthopaedic Trauma Society compared screw fixation (two, 3.5-mm, tricortical screws) vs TightRope; malreduction rates were lower with TightRope without finding differences in functional outcomes over time; as expected, reoperation rates were higher in the screw fixation group. ${ }^{53}$ Fan et al. published a systematic review and meta-analysis aiming to compare screw fixation with suture-button devices, 420 patients (10 studies) were included in the analysis. ${ }^{54}$ In their study, there were no statistically significant differences in functional outcomes, malreduction (although higher rates were found with screw fixation), and postoperative complications. A higher rate of secondary procedures, related to screw removal, was found. Literature has shown that postoperative outcomes, malreduction, and complication rates are similar between the two methods. ${ }^{54-56}$

\section{How Do I Assess My Reduction?}

Irrespective of the method used, it is important to achieve an adequate reduction of the syndesmosis as it affects fixation stability, ankle biomechanics, functional outcomes, and increases the risk of posttraumatic arthritis secondary to an increase in joint reactive forces. ${ }^{35,36,57-62}$ Malreduction has been identified in up 
to $50 \%$ of the cases and "small" differences $(2-\mathrm{mm})$ in the anterior TR distance when compared to the uninjured side showed to correlate with poorer outcomes. ${ }^{35,60,63,64}$ Persistent diastasis is often overlooked in plain radiographs, therefore the surgeon must be aware of the methods to evaluate diastasis intraoperatively and postoperatively. ${ }^{60}$

To evaluate instability intraoperatively, a hook test can be performed and can be complemented with an external rotation stress examination with fluoroscopic imaging. ${ }^{65}$ Even though these tests have a high interobserver agreement (99\%), it lacks sensitivity ( $25 \%$ and $58 \%$, respectively) to detect instability. ${ }^{66}$ Stress test can be complemented with a mortise and talar dome lateral fluoroscopic images of the uninjured side as a template for reduction and obtained an adequate reduction in 17/18 patients. ${ }^{67,68}$ Three-dimensional (3D) imaging has shown promising results and its use has been proposed as an alternative to identify subtle malreductions that are not identified in conventional fluoroscopy and/or postoperative radiographs. ${ }^{69}$ Franke et al. used intraoperative 3D scans to verify the reduction of 251 patients, almost a third of them required an improvement of the reduction. ${ }^{64}$ Despite this, literature has shown conflicting results while comparing 3D imaging to standard fluoroscopic images. ${ }^{70,71}$ It is important to remember that this method is not available in all hospital settings.

Postoperative CT scans have been used to evaluate reduction, Prior et al. published a protocol in which the anteroposterior translation and fibular length were measured and compared to the uninjured side. ${ }^{72}$ Abdelaziz et al. evaluated intraobserver and interobserver reliability of nine previously published methods used to evaluate reduction. Syndesmotic area calculation and fibular rotation proved to have the highest interobserver reliability. ${ }^{73}$

\section{Should I Remove My Implant?}

There seems to be an agreement regarding implant removal. Literature has shown that there is no additional benefit to screw removal as there is no difference in functional, clinical, or radiographic outcomes. ${ }^{74-76}$ It is important to have an early conversation with patients and explain that screw breakage is a possibility, more importantly, it is crucial to emphasize that this does not affect recovery nor outcomes, and hardware removal is not advisable. There are circumstances in which implant removal should be considered (e.g., persistent pain or syndesmosis malreduction). Early removal (i.e., 6-8 weeks) has been associated with syndesmotic diastasis. ${ }^{74,77}$ It is advisable to wait at least 12 weeks to guarantee adequate healing.

\section{ConcLusion}

Despite controversy and lack of consensus in some aspects of the diagnosis and treatment of syndesmotic lesions, irrespective of the reduction and fixation method used, to obtain good results it is essential to achieve an adequate reduction.

\section{References}

1. Coles CP, Tornetta P, Obremskey WT, et al. Ankle fractures: an expert survey of orthopaedic trauma association members and evidence-based treatment recommendations. J Orthop Trauma 2019;33(9):e318-e324. DOI: 10.1097/BOT.0000000000001503.

2. Van Heest TJ, Lafferty PM. Injuries to the ankle syndesmosis. J Bone Jt Surg - Ser A 2014;96(7):603-613. DOI: 10.2106/JBJS.M.00094.
3. Gougoulias N, Sakellariou A. When is a simple fracture of the lateral malleolus not so simple? Bone Jt J 2017;B(7):851-855. DOI: 10.1302/0301-620X.99B7.BJJ-2016-1087.R1.

4. DeAngelis NA, Eskander MS, French BG. Does medial tenderness predict deep deltoid ligament incompetence in supination-external rotation type ankle fractures? J Orthop Trauma 2007;21(4):244-247. DOI: 10.1097/BOT.0b013e3180413835.

5. Egol KA, Amirtharage M, Tejwani NC, et al. Ankle stress test for predicting the need for surgical fixation of isolated fibular fractures. J Bone Jt Surg - Ser A 2004;86(11):2393-2398. DOI: 10.2106/00004623200411000-00005.

6. Croft S, Furey A, Stone $C$, et al. Radiographic evaluation of the ankle syndesmosis. Can J Surg 2015;58(1):58-62. DOI: 10.1503/cjs.004214.

7. den Bekerom MPJ, Mutsaerts ELAR, Dijk CN. Evaluation of the integrity of the deltoid ligament in supination external rotation ankle fractures: a systematic review of the literature. Arch Orthop Trauma Surg 2009;129(2):227-235. DOI: 10.1007/s00402-008-0768-6.

8. Park SS, Kubiak EN, Egol KA, et al. Stress radiographs after ankle fracture: the effect of ankle position and deltoid ligament status on medial clear space measurements. J Orthop Trauma 2006;20(1):11-18. DOI: 10.1097/01.bot.0000189591.40267.09.

9. McConnell T, Creevy W, Tornetta P. Stress examination of supination external rotation-type fibular fractures. J Bone Jt Surg - Ser A 2004;86(10):2171-2178. DOI: 10.2106/00004623-200410000-00007.

10. Lambert LA, Falconer L, Mason L. Ankle stability in ankle fracture. J Clin Orthop Trauma 2020;11(3):375-379. DOI: 10.1016/j.jcot.2020.03.010.

11. Schock HJ, Pinzur M, Manion L, et al. The use of gravity or manualstress radiographs in the assessment of supination-external rotation fractures of the ankle. J Bone Jt Surg - Ser B 2007;89(8):1055-1059. DOI: 10.1302/0301-620X.89B8.19134.

12. Michelson JD, Varner KE, Checcone M. Diagnosing deltoid injury in ankle fractures. The gravity stress view. Clin Orthop Relat Res 2001. DOI: 10.1097/00003086-200106000-00024.

13. LeBa TB, Gugala Z, Morris RP, et al. Gravity versus manual external rotation stress view in evaluating ankle stability: a prospective study. Foot Ankle Spec 2015;8(3):175-179. DOI: 10.1177/1938640014565048.

14. Gill JB, Risko T, Raducan V, et al. Comparison of manual and gravity stress radiographs for the evaluation of supination-external rotation fibular fractures. J Bone Jt Surg - Ser A 2007;89(5):994-999. DOI: 10.2106/JBJS.F.01002.

15. Seidel A, Krause F, Weber M. Weightbearing vs gravity stress radiographs for stability evaluation of supination-external rotation fractures of the ankle. Foot Ankle Int 2017;38(7):736-744. DOI: $10.1177 / 1071100717702589$.

16. Saldua NS, Harris JF, LeClere LE, et al. Plantar flexion influences radiographic measurements of the ankle mortise. J Bone Jt Surg - Ser A 2010;92(4):911-915. DOI: 10.2106/JBJS.I.00084.

17. Weber $M$, Burmeister $H$, Flueckiger $G$, et al. The use of weightbearing radiographs to assess the stability of supination-external rotation fractures of the ankle. Arch Orthop Trauma Surg 2010;130(5):693-698. DOI: 10.1007/s00402-010-1051-1.

18. Hoshino CM, Nomoto EK, Norheim EP, et al. Correlation of weightbearing radiographs and stability of stress positive ankle fractures. Foot Ankle Int 2012;33(2):92-98. DOI: 10.3113/FAl.2012.0092.

19. Bäcker HC, Vosseller JT, Harald B, et al. Weightbearing radiography and MRI findings in ankle fractures. Foot Ankle Spec 2020. DOI: 10.1177/1938640020921571.

20. Brown KW, Morrison WB, Schweitzer ME, et al. MRI findings associated with distal tibiofibular syndesmosis injury. Am J Roentgenol 2004;182(1):131-136. DOI: 10.2214/ajr.182.1.1820131.

21. Park YH, Yoon MA, Choi WS, et al. The predictive value of MRI in the syndesmotic instability of ankle fracture. Skeletal Radiol 2018;47(4):533-540. DOI: 10.1007/s00256-017-2821-4.

22. Großterlinden LG, Hartel M, Yamamura J, et al. Isolated syndesmotic injuries in acute ankle sprains: diagnostic significance of clinical examination and MRI. Knee Surgery, Sport Traumatol Arthrosc 2016;24(4):1180-1186. DOI: 10.1007/s00167-015-3604-x. 
23. Oae K, Takao M, Naito K, et al. Injury of the tibiofibular syndesmosis: value of MR imaging for diagnosis. Radiology 2003;227(1):155-261. DOI: 10.1148/radiol.2271011865.

24. Nortunen S, Lepojärvi S, Savola O, et al. Stability assessment of the ankle mortise in supination-external rotation-type ankle fractures: lack of additional diagnostic value of MRI. J Bone Jt Surg - Am 2014;96(22):1855-1862. DOI: 10.2106/JBJS.M.01533.

25. Warner SJ, Garner MR, Fabricant PD, et al. The diagnostic accuracy of radiographs and magnetic resonance imaging in predicting deltoid ligament ruptures in ankle fractures. HSS J 2019;15(2):115-121. DOI: 10.1007/s11420-018-09655-x.

26. Jenkinson RJ, Sanders DW, Macleod MD, et al. Intraoperative diagnosis of syndesmosis injuries in external rotation ankle fractures. J Orthop Trauma 2005;19(9):604-609. DOI: 10.1097/01. bot.0000177114.13263.12.

27. Boszczyk A, Kwapisz S, Krümmel M, et al. Correlation of incisura anatomy with syndesmotic malreduction. Foot Ankle Int 2018;39(3):369-375. DOI: 10.1177/1071100717744332.

28. Phisitkul $P$, Ebinger $T$, Goetz J, et al. Forceps reduction of the syndesmosis in rotational ankle fractures: a cadaveric study. J Bone Jt Surg - Ser A 2012;94(24):2256-2261. DOI: 10.2106/JBJS.K.01726.

29. Rammelt S, Obruba P. An update on the evaluation and treatment of syndesmotic injuries. Eur J Trauma Emerg Surg 2015;41(6):601-614. DOI: 10.1007/s00068-014-0466-8.

30. Cosgrove CT, Putnam SM, Cherney SM, et al. Medial clamp tine positioning affects ankle syndesmosis malreduction. J Orthop Trauma 2017;31(8):440-446. DOI: 10.1097/BOT.0000000000000882.

31. Cosgrove CT, Spraggs-Hughes AG, Putnam SM, et al. A novel indirect reduction technique in ankle syndesmotic injuries: a cadaveric study. J Orthop Trauma 2018;32(7):361-367. DOI: 10.1097/ BOT.0000000000001169.

32. Putnam SM, Linn MS, Spraggs-Hughes A, et al. Simulating clamp placement across the trans-syndesmotic angle of the ankle to minimize malreduction: a radiological study. Injury 2017;48(3):770775. DOI: 10.1016/j.injury.2017.01.029.

33. Vetter SY, Beisemann N, Keil $\mathrm{H}$, et al. Comparison of three different reduction methods of the ankle mortise in unstable syndesmotic injuries. Sci Rep 2019;9(1). DOI: 10.1038/s41598-019-51988-y.

34. Park YH, Ahn JH, Choi GW, et al. Comparison of clamp reduction and manual reduction of syndesmosis in rotational ankle fractures: a prospective randomized trial. J Foot Ankle Surg 2018;57(1):19-22. DOI: 10.1053/j.jfas.2017.05.040.

35. Sagi HC, Shah AR, Sanders RW. The functional consequence of syndesmotic joint malreduction at a minimum 2-year follow-up. J Orthop Trauma 2012;26(7):439-443. DOI: 10.1097/ BOT.0b013e31822a526a.

36. Miller AN, Carroll EA, Parker RJ, et al. Direct visualization for syndesmotic stabilization of ankle fractures. Foot Ankle Int 2009;30(5):419-426. DOI: 10.3113/fai.2009.0419.

37. Pelton $K$, Thordarson DB, Barnwell J. Open versus closed treatment of the fibula in maissoneuve injuries. Foot Ankle Int 2010;31(7):604-608. DOI: 10.3113/FAI.2010.0604.

38. Tornetta $\mathrm{P}$, Yakavonis M, Veltre $\mathrm{D}$, et al. Reducing the syndesmosis under direct vision: where should I look? J Orthop Trauma 2019;33(9):450-454. DOI: 10.1097/BOT.0000000000001552.

39. Miller MA, McDonald TC, Graves ML, et al. Stability of the syndesmosis after posterior malleolar fracture fixation. Foot Ankle Int 2018;39(1):99-104. DOI: 10.1177/1071100717735839.

40. Li M, Collier RC, Hill BW, et al. Comparing different surgical techniques for addressing the posterior malleolus in supination external rotation ankle fractures and the need for syndesmotic screw fixation. J Foot Ankle Surg 2017;56(4):730-734. DOI: 10.1053/j.jfas.2017.01.053.

41. Langenhuijsen JF, Heetveld MJ, Ultee JM, et al. Results of ankle fractures with involvement of the posterior tibial margin. J Trauma 2002;53(1):55-60. DOI: 10.1097/00005373-200207000-00012.

42. Hansen M, Le L, Wertheimer $S$, et al. Syndesmosis fixation: analysis of shear stress via axial load on $3.5-\mathrm{mM}$ and $4.5-\mathrm{mM}$ quadricortical syndesmotic screws. J Foot Ankle Surg 2006;45(2):65-69. DOI: 10.1053/j.jfas.2005.12.004.

43. Markolf KL, Jackson SR, McAllister DR. Syndesmosis fixation using dual $3.5 \mathrm{~mm}$ and $4.5 \mathrm{~mm}$ screws with tricortical and quadricortical purchase: a biomechanical study. Foot Ankle Int 2013;34(5):734-739. DOI: 10.1177/1071100713478923.

44. Thompson MC, GesinkDS. Biomechenical comparison of syndesmosis fixation with 3.5- and 4.5-millimeter stainless steel screws. Foot Ankle Int 2000;21(9):736-741. DOI: 10.1177/107110070002100904.

45. Beumer A, Campo MM, Niesing R, et al. Screw fixation of the syndesmosis: a cadaver model comparing stainless steel and titanium screws and three and four cortical fixation. Injury 2005;36(1):60-64. DOI: 10.1016/j.injury.2004.05.024.

46. Peek AC, Fitzgerald CE, Charalambides C. Syndesmosis screws: how many, what diameter, where and should they be removed? A literature review. Injury 2014;45(8):1262-1267. DOI: 10.1016/j. injury.2014.05.003.

47. Høiness P, Strømsøe K. Tricortical versus quadricortical syndesmosis fixation in ankle fractures: a prospective, randomized study comparing two methods of syndesmosis fixation. J Orthop Trauma 2004;18(6):331-337. DOI: 10.1097/00005131-200407000-00001.

48. Wikerøy AKB, Høiness PR, Andreassen GS, et al. No difference in functional and radiographic results 8.4 years after quadricortical compared with tricortical syndesmosis fixation in ankle fractures. J Orthop Trauma 2010;24(1):17-23. DOI: 10.1097/BOT.0b013 e3181bedca1.

49. Moore JA, Shank JR, Morgan SJ, et al. Syndesmosis fixation: a comparison of three and four cortices of screw fixation without hardware removal. Foot Ankle Int 2006;27(8):567-572. DOI: 10.1177/107110070602700801.

50. Kukreti S, Faraj A, Miles JNV. Does position of syndesmotic screw affect functional and radiological outcome in ankle fractures? Injury 2005;36(9):1121-1124. DOI: 10.1016/j.injury.2005.01.014.

51. Lee JS, Curnutte B, Pan K, et al. Biomechanical comparison of suturebutton, bioabsorbable screw, and metal screw for ankle syndesmotic repair: a meta-analysis. Foot Ankle Surg 2020. S1268-7731(20)30046-1 10.1016/j.fas.2020.03.008.

52. Parker AS, Beason DP, Slowik JS, et al. Biomechanical comparison of 3 syndesmosis repair techniques with suture button implants. Orthop J Sport Med 2018;6(10):2325967118804204 10.1177/2325967118804204.

53. Sanders D, Schneider P, Taylor M, et al. Improved reduction of the tibiofibular syndesmosis with tightrope compared with screw fixation: results of a randomized controlled study. J Orthop Trauma 2019;33(11):531-537. DOI: 10.1097/BOT.0000000000001559.

54. Fan $X$, Zheng $P$, Zhang $Y-Y$, et al. Dynamic fixation versus static fixation in treatment effectiveness and safety for distal tibiofibular syndesmosis injuries: a systematic review and meta-analysis. Orthop Surg 2019;11(6):923-931. DOI: 10.1111/os.12523.

55. Kortekangas T, Savola O, Flinkkilä T, et al. A prospective randomised study comparing tightrope and syndesmotic screw fixation for accuracy and maintenance of syndesmotic reduction assessed with bilateral computed tomography. Injury 2015;46(6):1119-1126. DOI: 10.1016/j.injury.2015.02.004.

56. Zhang $P$, Liang $Y, H e J$, et al. A systematic review of suture-button versus syndesmotic screw in the treatment of distal tibiofibular syndesmosis injury. BMC Musculoskelet Disord 2017;18(1):286. DOI: 10.1186/s12891-017-1645-7.

57. Bai L, Zhang W, Guan S, et al. Syndesmotic malreduction may decrease fixation stability: a biomechanical study. J Orthop Surg Res 2020;15(1). DOI: 10.1186/s13018-020-01584-y.

58. Miller AN, Barei DP, laquinto JM, et al. latrogenic syndesmosis malreduction via clamp and screw placement. J Orthop Trauma 2013;27(2):100-106. DOI: 10.1097/BOT.0b013e31825197cb.

59. Rushing CJ, Spinner SM, Armstrong AV, et al. Comparison of different magnitudes of applied syndesmotic clamp force: a cadaveric study. J Foot Ankle Surg 2020;59(3):452-456. DOI: 10.1053/j.jfas.2019. 08.028 . 
60. Gardner MJ, Demetrakopoulos D, Briggs SM, et al. Malreduction of the tibiofibular syndesmosis in ankle fractures. Foot Ankle Int 2006;27(10):788-792. DOI: 10.1177/107110070602701005.

61. Weening B, Bhandari M. Predictors of functional outcome following transsyndesmotic screw fixation of ankle fractures. J Orthop Trauma 2005;19(2):102-108. DOI: 10.1097/00005131-200502000-00006.

62. Van Vlijmen N, Denk K, Van Kampen A, et al. Long-term results after ankle syndesmosis injuries. Orthopedics 2015;38(11):e1001-e1006. DOI: 10.3928/01477447-20151020-09.

63. Andersen MR, Diep LM, Frihagen F, et al. Importance of syndesmotic reduction on clinical outcome after syndesmosis injuries. J Orthop Trauma 2019:33(8):397-403. DOI: 10.1097/BOT.0000000000001485.

64. Franke J, Von Recum J, Suda AJ, et al. Intraoperative three-dimensional imaging in the treatment of acute unstable syndesmotic injuries. J Bone Jt Surg - Ser A 2012;94(15):1386-1390. DOI: 10.2106/JBJS.K. 01122.

65. Stark E, Tornetta $P$, Creevy WR. Syndesmotic instability in weber B ankle fractures: a clinical evaluation. J Orthop Trauma 2007;21(9):643646. DOI: 10.1097/BOT.0b013e318157a63a.

66. Pakarinen $\mathrm{H}$, Flinkkilä $\mathrm{T}$, Ohtonen $\mathrm{P}$, et al. Intraoperative assessment of the stability of the distal tibiofibular joint in supination-external rotation injuries of the ankle sensitivity, specificity, and reliability of two clinical tests. J Bone Jt Surg - Ser A 2011;93(22):2057-2061. DOI: 10.2106/JBJS.J.01287.

67. Summers HD, Sinclair MK, Stover MD. A reliable method for intraoperative evaluation of syndesmotic reduction. J Orthop Trauma 2013;27(4):196-200. DOI: 10.1097/BOT.0b013e3182694766.

68. Schreiber JJ, McLawhorn AS, Dy CJ, et al. Intraoperative contralateral view for assessing accurate syndesmosis reduction. Orthopedics 2013:36(5):360-361. DOI: 10.3928/01477447-20130426-03.
69. Dubois-Ferrière V, Gamulin A, Chowdhary A, et al. Syndesmosis reduction by computer-assisted orthopaedic surgery with navigation: Feasibility and accuracy in a cadaveric study. Injury 2016;47(12):2694-2699. DOI: 10.1016/j.injury.2016.10.009.

70. Ruan Z, Luo C, Shi Z, et al. Intraoperative reduction of distal tibiofibular joint aided by three-dimensional fluoroscopy. Technol Heal Care 2011;19(3):161-166. DOI: 10.3233/THC-2011-0618.

71. Davidovitch RI, Weil Y, Karia R, et al. Intraoperative syndesmotic reduction: three-dimensional versus standard fluoroscopic imaging. J Bone Jt Surg - Ser A 2013;95(20):1838-1843. DOI: 10.2106/ JBJS.L.00382.

72. Prior CP, Widnall JC, Rehman AK, et al. A simplified, validated protocol for measuring fibular reduction on ankle CT. Foot Ankle Surg 2017;23(1):53-56. DOI: 10.1016/j.fas.2016.02.005.

73. Abdelaziz ME, Hagemeijer N, Guss D, et al. Evaluation of syndesmosis reduction on CT scan. Foot Ankle Int 2019;40(9):1087-1093. DOI: 10.1177/1071100719849850.

74. Walley KC, Hofmann KJ, Velasco BT, et al. Removal of hardware after syndesmotic screw fixation: a systematic literature review. Foot Ankle Spec 2017;10(3):252-257. DOI: 10.1177/1938640016685153.

75. DIngemans SA, Rammelt S, White TO, et al. Should syndesmotic screws be removed after surgical fixation of unstable ankle fractures? a systematic review. Bone Jt J 2016;98-B(11):1497-1504. DOI: 10.1302/0301-620X.98B11.BJJ-2016-0202.R1.

76. Gennis E, Koenig S, Rodericks D, et al. The fate of the fixed syndesmosis over time. Foot Ankle Int 2015;36(10):1202-1208. DOI: $10.1177 / 1071100715588186$.

77. Pogliacomi F, Artoni C, Riccoboni S, et al. The management of syndesmotic screw in ankle fractures. Acta Biomed 2019;90(1): 146-149. 